\title{
Learning by e-Learning: Breaking Down Barriers and Creating Opportunities for the Visually-Impaired
}

\author{
Barbara Leporini ${ }^{1}$ and Marina Buzzi ${ }^{2}$ \\ ${ }^{1}$ CNR-ISTI, via Moruzzi 1, 56124, Pisa, Italy \\ barbara.leporini@isti.cnr.it \\ ${ }^{2}$ CNR-IIT, via Moruzzi 1, 56124 Pisa, Italy \\ marina.buzzi@iit.cnr.it
}

\begin{abstract}
Today e-Learning is an important educational tool with multiple benefits. However, to be suitable for a large audience the learning objects, courses, and other forms of e-Learning content should be easy to use for all students, regardless of disability. This paper describes problems often encountered by a blind person using screen reader and voice synthesizer when using e-Learning systems, and proposes guidelines for designers in order to develop more accessible systems.
\end{abstract}

Keywords: Accessibility, usability, e-Learning, blind.

\section{Introduction}

In recent years e-Learning has become a valuable tool for an increasing number of users. The benefits of this technology include (I) remote learning for students, (II) remote teaching for teachers living far from schools or universities, III) continuing education for adults.

A number of studies (e.g. [3], [11]) confirm that students appreciate the advantages of electronic materials in terms of portability and overall ubiquity, but they also value legibility, presentation and good design. There is also a clear demand for extra functions such as smart searches and dynamic indexing. Quality and the ability to provide extra facilities unavailable in paper textbooks are crucial for the future of electronic learning materials.

Distance learning can be a valuable opportunity for the visually-impaired and for other categories of disabled users, if suitable education methods and appropriate technologies are used. In particular, when a learning object or an e-Learning platform is accessible to but not usable by blind users, the benefits of distance learning methodology risk being worthless for them. Hence, when designing electronic learning materials (i.e. Learning Objects) and delivery systems (e.g. Learning Management Systems) the main issue concerns the users' abilities: it is crucial to identify the needs and requirements of the target community to create a system that fulfills their expectations. Thus a well-defined user profile is an essential component of the design process for the successful development of e-Learning Systems as well as Learning Objects. 
In this preliminary phase of our research we analyzed user requirements and provide an overview of key aspects for the visually-impaired, highlighting unresolved issues and identifying future perspectives. It does not pretend to be an exhaustive analysis, but this initial investigation aims to provide an overview of the subject concerning people with a visual disability.

The paper is organized as follows: first we give an overview of studies in this field. Next we describe the main problems encountered by visually-impaired individuals when interacting by screen reader. We then discuss basic accessibility and usability issues related to e-Learning methodology and systems.

\section{Related Works}

In the last few years e-Learning technology has become an important research topic. Design and usability of e-Learning systems and objects is a primary focus of research in this field. However, interactive learning is still very difficult for blind persons who use assistive technologies such as screen readers and voice synthesizers. Various studies focus on the usability of e-Learning systems and some also include a general discussion on accessibility, but to our knowledge only a few specifically involve a study concerning the blind.

E-learning systems pose new challenges with respect to User Centered Design, where the target is a set of homogeneous users. Learner Centered Design must answer to the needs of multiple learner categories due to differences in learning strategies, know-how, experience in the learning domain, motivation to learn and user abilities.

In 1994 Nielsen proposed an informal method for evaluating systems and design usability based on a set of principles of usable software design (heuristics) [8]. A group of experts looks for violations of these heuristics, and thus the problems identified (a high percentage of the total) are fixed before the product arrives at the user. This method is cost-effective and easy to carry out compared to usability testing or other evaluation techniques. However, these principles were general, and in order to be applied to e-Learning applications and systems needed to be adapted to the specific domain. Squires and Preece in 1999 filtered usability heuristics throughout the socio-constructive theory and specified criteria ad hoc for e-Learning [10]. However various researchers argue the lack of accurate studies in this field. In [2] the authors take the first steps in defining a methodology for the rigorous evaluation of eLearning applications, but accessibility for disabled students is not analyzed. Furthermore, Zaharias critically examined the usability of e-Learning applications and proposed a new usability measure: the student's intrinsic motivation to learn [12]. Developing a usability evaluation method based on a questionnaire he carried out two large empirical studies that showed the reliability of this approach. However, since it is a work in progress, details are not available regarding the accessibility section of the questionnaire.

Sloan et al. highlight limits and failures of the Web Content Accessibility Guidelines and propose treating accessibility with a holistic approach [9]. They believe that the goal of universal accessibility on the Web is inappropriate and that instead it is necessary to explore multiple routes to equivalent experience. As Kelly et al. argued, rather than demanding that an individual learning resource be universally 
accessible, it is the learning outcome that needs to be accessible [7]. Based on user profiles, metadata and dynamic connection to resources, the experience of the user may be customized to match his/her abilities. We personally agree with this point of view and we believe that appropriate design is crucial for improving the accessibility and usability of e-Learning Systems. In [1] the study offers an example of designing UIs for the blind, applied to search engines.

Last, De Marsico et al. [5] define methodological guidelines of a joint design framework based on involving users with disabilities, as well as pedagogical experts, in the development process, believing that input of different know-how may enrich the quality of e-Learning applications, and provide a more satisfying learning experience. They also include two examples of building and providing learning objects accessible respectively to visually- (first example) and hearing- impaired students (second example).

Although the paper focuses on the needs of blind users, all disabilities should be considered when designing e-Learning applications. The criteria proposed ad-hoc for the blind must be integrated with others design guidelines. For example delivery of audio content should provide text and graphical content to supplement the audio channel (taking care to keep the content synchronized throughout the various media) and captions should be provided for any video incorporated into e-Learning applications.

\section{Interacting Via Screen Reader and Voice Synthesizer}

Designers of e-Learning systems must consider three crucial factors: usability, accessibility, and educational effectiveness. Consequently, the challenge is to design systems that are accessible to everyone and simple to use while maintaining pedagogical and educational efficacy. In particular, blind students may fruitfully utilize e-Learning systems if educational materials are accessible and learning paths can be tuned to the "rhythm" of the individual student.

When designing for blind users, it is necessary to consider the three main interacting subsystems of the Human Processor Model: the perceptual, motor and cognitive systems [4]. Sightless persons perceive page content aurally and do not interact with the mouse or other pointing devices since they only navigate via keyboard. This makes the "reading process" time-consuming and sometimes difficult and frustrating, if the contents are not designed with special attention to their needs.

The cognition part of the interaction is important, since many learning techniques are only relevant to people with good vision and may not apply to someone with a visual impairment. Thus alternative ways to deliver the same content should be provided. Furthermore, a blind person may develop a different mental model of both the interaction and the learning processes thus it is crucial to provide an easy overview of the system and contents.

Specifically non-visual perception can lead to many issues:

1. Content serialization. The screen reader reads the contents sequentially, as they appear in the HTML code. This process is time-consuming and annoying when part of the interface (e.g. the menu and/or the navigation bar) is repeated in many pages of the UI. As a consequence, blind users often have to stop the screen 
reading at the beginning, and they prefer to navigate by Tab Keys, from link to link, or explore the content row by row, via arrow keys.

2. Content and structure mixing. The screen reader announces the most important interface elements such as links, images, and window objects as they appear in the code. For the blind user, these elements are important for figuring out the page structure. However, the actual reading process can overload the user, requiring considerable cognitive effort. If the table's content is organized by columns the screen reader (which reads by rows) announces the content of the page out-oforder, and consequently the information is confusing or misleading for the user. For the same reason, the table used for the layout must be avoided.

3. Lack of context. When navigating by screen reader the user can access only small portions of text and may lose the overall context of the page; thus it may be necessary to reiterate the reading process.

4. Lack of interface overview. Blind persons do not perceive the overall structure of the interface, so they can navigate for a long time without finding the most relevant contents. To resolve this problem it is necessary to structure the HTML code appropriately, add hidden information, and apply other features to improve navigation.

5. Difficulty understanding UI elements. Links, content, and button labels should be context-independent and self-explanatory.

6. Difficulty working with form control elements. The screen reader can handle the form elements by activating the modality - "form mode on" (e.g. by pressing the Enter key when the virtual focus is over a form control). In this situation, the screen reader informs the user by reading the sentence "form mode on". If the user forgets to switch on this modality, no typed text is written in a text field, no choice is selected from a combo-box, and some unwanted effects may occur since in modality "form mode off" any key pressure performs a different command (e.g. " $t$ " moves to the following table and " $h$ " to the following heading). Consequently, the user needs to re-start the operation.

7. Last, obviously, a blind person is unable to access multimedia content such as video streaming, video conferencing, and captioning.

\section{Accessibility and Usability Issues in e-Learning}

In order to create a distance learning course for all, the e-Learning system itself as well as the content should be adequate to users' abilities. E-Learning (literally electronic learning) is an all-encompassing term generally used to refer to computerenhanced learning. It may include the use of web-based teaching materials and hypermedia in general, multimedia CD-ROMs or web sites, discussion boards, collaborative software, e-mail, blogs, wikis, text chat, computer aided assessment, educational animation, simulations, games, learning management software, electronic voting systems and more, as well as a combination of different methods. A particular contribution to the learning process in such an interactive environment is provided by 
collaborative and cooperative technologies. For instance, the increasing trend is to create a Virtual Learning Environment (VLE) where students can collaborate and cooperate. Various studies to investigate the real effectiveness and efficiency of such environments have been conducted [6].

Pedagogically speaking, we should recall that the learning process is the main focus of an e-Learning-based education methodology. Students should be able to concentrate on contents and educational activities without being distracted by the system. That means that if all components are not accessible and suited to the user's capabilities, difficulties interacting with the system could distract the student from learning.

An e-Learning environment is basically composed of two main parts: (I) the integrated system - i.e. the container, which provides the contents and interactive activities to the participants; (II) the Learning Object (LO) - the educational contents provided through the e-Learning interactive systems.

Consequently, when designing an e-Learning environment suitable for various users including those with disabilities, two main aspects should be kept in mind:

- Accessibility and usability of the system, i.e. the "container" which provides contents and activities for the participants should be easy to use for all;

- Accessibility and usability of contents, that is the texts, documents and interactive tools used for educational purposes should be created according to various users' abilities.

If either of the two aspects is not accessible and usable, interactive distance education methodology risks being ineffective for people who are obliged to interact with assistive technology. This is particularly significant for blind users who interact by screen reader, which interprets interface content sequentially. Thus, it is important to consider both aspects when designing and developing a distance course.

\subsection{Learning Management Systems}

A Learning Management System (or LMS) is a software package that enables the management and delivery of on-line content to learners. Most LMSs are web-based to facilitate "anytime, any place, any pace" access to learning content and administration. Typically an LMS permits learner registration, delivery of learning activities, and learner assessment in an on-line environment. Both commercial and free platforms are developed. Open source and Web-based LMS software solutions are growing rapidly in the education and business worlds.

In addition to managing the administrative functions of on-line learning, Learning Management Systems also provide tools for delivering instructor-led synchronous and asynchronous on-line training (Learning Content Management Systems). An LCMS provides tools for authoring content as well as virtual spaces for learner interaction (such as discussion forums and live chat rooms).

Often, when considering accessibility issues only disabled students are considered, thus only LMSs are taken into account. Instead, people with disabilities may also be teachers, who must be able to create and manage the materials to use for distance courses. Thus LCMS authoring functionalities should also be accessible and usable by assistive technologies. In the following we generalize the discussion concerning 
accessibility and usability features referring to both kinds of platforms (LMS and LCMS).

Since the user interface of an e-Learning system is usually based on Web technologies, at first glance it may appear sufficient to apply web accessibility guidelines. Actually, regarding the most widespread e-Learning platforms, some considerations can be made regarding technical aspects:

- Student tracking: provides information about the individual use patterns of the students in the course. Scorm-based platforms use javascript technologies for tracking the activities. For accessibility principles, the platform should offer the same functions when javascripts are disabled as well. Since most e-Learning systems - such as moodle (http://moodle.org/), atutor (http://www.atutor.ca/), and so on - are scorm-based, javascript is used. Alternative implementations should be investigated.

- Hidden frames: several e-Learning platforms use hidden frames for storing data to communicate to the Web server. Although for usability principles frames should not be used, appropriate design suggests using few hidden frames and positioning them after the frames containing the learning objects. In order to further simplify interaction, it is useful to give appropriate names to all the frames.

\subsection{Learning Objects}

A Learning Object (LO) may be defined as any entity, digital or non-digital, that can be used for learning, education or training (IEEE/LTSC). A Learning Object can be as small as a paragraph or as large as a complete online course and come in the form of HTML/Text files, simulations, JAVA, Flash, QuickTime movies etc. According to the format and complexity of a LO, various guidelines and principles should be applied to make them accessible and usable as well.

Apart from technical accessibility, we think that a specific issue should be considered when developing a Learning Object. Since the main goal of e-Learning methodology is education, a LO should be designed to exploit the user's abilities. For instance, for a sighted child an image or animation is probably more effective than a descriptive LO whereas for a blind child an audio object is much more effective than a textual one. Thus, the way a Learning Object is designed and structured is very important. In this case it could be made more effective by using different LOs for different users' abilities and skills. Further study is necessary in order to define a more appropriate approach for developing accessible and usable LOs.

Moreover, we think that most difficulties arise from simulation activities. Simulation systems and LOs can have various technical accessibility problems. A target study in this direction could prove useful.

\section{Design Criteria}

To make e-Learning systems accessible and usable, both the interactive system (Learning Management System) and the contents (Learning Objects) must be adequately developed. To this end, appropriate guidelines, requirements and suggestions can help developers keep the main issues in mind. 
When designing an e-Learning system, the analyst needs to consider that in order to move quickly around the page content, the blind person prefers to use the Tab key to jump from one interactive element to another, rather than explore content via arrow keys.

Furthermore, objective difficulties may be reduced or eliminated by presenting the same content in aural form and providing multiple ways to navigate faster.

Last, when removing technical barriers for accessing contents, it is important to simplify user interaction in order to make the learning process more effective and satisfying.

\subsection{Interface Overview}

First of all, the blind person does not perceive the overall structure of the interface. Therefore it is important to structure the HTML code, defining logical sections of the interface.

Specifically, it is possible to group and structure (by heading levels) sets of homogenous text and elements in order to give the user immediately the idea of the interface, and enable him to jump rapidly from one section to another. For example, heading tags $(<\mathrm{h} 1>$.. $<\mathrm{h} 6>)$, if appropriately applied, are particularly useful since they are captured by screen reader and listed as an index, helping the user to "navigate" the interface.

Furthermore the use of some hidden information could help users to better comprehend the page structure.

\subsection{Order of Content Blocks}

Important content should be placed at the beginning of the interface code (i.e. the xhtml or html file) in order to facilitate sequential reading. Indeed, the position of the most important content is crucial for a blind user who usually wishes to explore the least amount of information necessary. Navigation of the container may become an obstacle to learning, so it is very important to place the most important element of the interface at the top of the content read by screen reader in order to access relevant content quickly. This feature was the most appreciated by totally blind users in our previous work [1]. The graphical interface instead must conform to the usual HCI criteria, arranging the content in a more appropriate way for visual elaboration.

\subsection{Quick Comprehension of the Most Important Events}

Aural feedback is very useful for the blind user since it associates a specific sound with a given situation (e.g. different tones may be associated with the success or failure of an operation) and simplifies interaction with form control elements.

The designer may include this feature in the e-Learning system interfaces, adding tones in some cases, such as:

- When the interactive form elements (edit field, radio button, or checkbox) receives the focus;

- When a lesson module is finished or a new module is started;

- To immediately inform the user of the success or failure of an operation (for instance the result of a self-assessment). 


\subsection{Simple Keyboard Interaction}

Interaction with the system via keyboard is simplified if shortcuts and navigation via Tab keys are provided in order to jump directly to the most important elements of the UIs.

- Access keys must be associated with the most important interface elements offering a simple shortcut to the desired point (e.g. skip to the beginning of module, go to course index, move on evaluation assessment, and so on).

- Tab Keys define an order of visit of UI elements (e.g. links, objects). In this way, the order of "importance" associated with each element of the interface (i.e. the value of the tab access attribute) "drives" the user's navigation by Tab key. A lower tab-index value indicates greater importance.

\subsection{Multimedia}

Inability to access multimedia content such as video streaming, video conferencing, and captioning may be partially resolved by providing text equivalents, readable by screen reader. For example, supplementary content in an audio presentation can include the exact text presented as well as the description of relevant graphical content.

\section{Discussion}

E-Learning methodology can be an excellent educational opportunity for users with disabilities. A distance course offers students advantages such as flexibility and convenience, facilitation of communication between learners, greater adaptability to a learner's needs, more variety in learning experience with the use of multimedia and the non-verbal presentation of teaching material. Video instruction provides visual and audio learning that can be paused and reversed for watching again. Ability to access materials and the virtual environment both synchronously and asynchronously is a good opportunity for people who have difficulties in terms of time or space. This is particularly true for visually-impaired individuals who may have problems with classroom learning.

E-Learning in general education has been criticized since face-to-face human interaction with a teacher has been removed from the process; thus, some argue, the process is no longer "educational" in the highest philosophical sense. A blended methodology may be much more effective and efficient in terms of educational purposes, as observed by one of the authors of this paper in courses for blind students. Interacting with an e-Learning system is more complicated for a blind user than for a user with no visual impairment since interaction requires an assistive technology, which adds a level of complexity; thus an initial face-to-face phase may be necessary. Apart from this, e-Learning methodology represents a great opportunity for visuallydisabled people, provided the interactive system is properly designed and developed.

The feeling of isolation experienced by distance learning students is often cited as a negative aspect, although discussion forums and other computer-based communication 
can help overcome this and often encourage students to meet face-to-face and form selfhelp groups.

In the personal experience of this paper's blind author, we observed that blind users greatly appreciate mailing lists and, to a lesser degree, on-line forums. This is probably due to the fact that mailing lists have a sequential access; instead the forum interaction via Web interface may present usability problems.

\section{Conclusions}

In this preliminary work we have discussed accessibility issues for e-Learning systems (LMSs and LOs) and have proposed empirical principles for designers developing e-Learning applications in order to simplify interaction for a blind student or teacher.

We argued that both LMSs and LOs should be appropriately designed. E-Learning environments should be friendly and easy to use in order to reduce any possible negative effects on the learning process. However, rendering LMSs and LOs suitable for the abilities and skills of all users presents many challenges.

When defining the GUI it is fundamental to consider the needs of sighted users but the needs of blind users should also be taken into account when writing the UI code; furthermore the same information should be provided through both visual and auditory channels.

In conclusion, further research in this direction is needed. Future studies will investigate the empirical adaptability of an e-Learning system as well as suitable Learning Objects. Moreover, additional features of an LMS such as adaptability and customization will be addressed.

\section{References}

1. Andronico, P., Buzzi, M., Castillo, C., Leporini, B.: Improving Search Engine Interfaces for Blind Users: a Case Study. Springer UAIS Journal 5(1), 23-40 (2006)

2. Ardito, C., De Marsico, M., Lanzilotti, R., Levialdi, S., Roselli, T., Rossano, V., Tersigni, M.: Usability of e-Learning tools. In: Proceedings of the working conference on Advanced visual interfaces (2004)

3. Ardito, C., Costabile, M., De Marsico, M., Lanzilotti, R., Levialdi, S., Roselli, T., Rossano, V.: An Approach to Usability Evaluation of e-Learning Applications. Universal Access In. the Information Society 4(3), 270-283 (2005)

4. Card, S.K., Moran, A., Newell, T.P.: The Psychology of Human-Computer Interaction. Lawrence Erlbaum Associates Inc, New Jersey (1983)

5. De Marsico, M., Kimani, S., Mirabella, V., Norman, K.L., Catarci, T.: A proposal toward the development of accessible e-Learning content by human involvement. Springer, UAIS Journal 5(2), 150-169 (2006)

6. Eijl, P., Pilot, A., Voogd, P.: Effects of Collaborative and Individual Learning in a Blended Learning Environment. Education and Information Technologies 10(1-2), 51-65 (2005) 
7. Kelly, B., Phipps, L., Howell, C.: Implementing a holistic approach to e-Learning accessibility. Retrieved at http://www.ukoln.ac.uk/web-focus/papers/alt-c2005/accessibility-elearning-paper.doc

8. Nielsen, J.: Usability inspection methods. In: Heuristic evaluation, pp. 25-62. John Wiley \& Sons, Inc, New York, USA (1994)

9. Sloan, D., Heath, A., Hamilton, F., Kelly, B., Petrie, H., Phipp, L.: Contextual web accessibility - maximizing the benefit of accessibility guidelines. In: Proceedings of the 2006 international cross-disciplinary workshop on Web accessibility (2006)

10. Squires, D., Preece, J.: Predicting quality in educational software: Evaluating for learning, usability and the synergy between them. Interacting with Computers 11(5), 467-483 (1999)

11. Wilson, R., Landoni, M., Gibb, F.: A user-centered approach to e-book design. The Electronic Library, vol. 20(4) (2002)

12. Zaharias, P.: A usability evaluation method for e-learning: focus on motivation to learn. In: Proceedings of CHI '06 extended abstracts on Human factors in computing systems (2006) 